\title{
Delivering bad news by physicians - Polish reality check
}

\author{
Krzysztof Sobczak', Leszek Pawłowski², Małgorzata Pietrzykowska ${ }^{3}$, Natalia Spolak ${ }^{4}$ \\ 1 Department of Social Medicine and Social Pathology, Medical University of Gdansk, Gdansk, Poland \\ ${ }^{2}$ Department of Palliative Medicine, Medical University of Gdansk, Gdansk, Poland \\ ${ }^{3}$ Department of Family Medicine, Medical University of Gdansk, Gdansk, Poland \\ ${ }^{4}$ Department of Allergology in Medical University of Gdansk and University Clinical Centre in Gdansk, Gdansk, Poland
}

\begin{abstract}
Introduction. Disclosing unfavorable information is a very important moment in both diagnostic and therapeutic processes. It is also a highly stress-inducing factor, both among patients and physicians. During our research we tried to establish how exactly bad news is communicated to patients and the amount of stress that Polish physicians are under in such situations.

Material and Methods. Quantitative research was conducted in a university clinical hospital. With the use of an anonymous questionnaire, physicians $(n=100)$ from oncology, internal diseases, cardiac surgery, gynecology, obstetrics, and urology clinics were asked about the sources and the intensity of stress involved in BBN (Breaking Bad News). Similarly, patients $(n=378)$ of said clinics were asked to evaluate the relationships they had with their doctors.

Results. Most (66.7\%) clinicians declared they always conveyed unfavorable information to their patients fully and in detail. Exactly $50.0 \%$ admitted they were experiencing high or very high level of stress while doing so. They were mostly (56.1\%) anxious about depriving their patients of hope and (38.5\%) feared they were letting their patients down. $37.3 \%$ of clinicians were afraid of emotional response. Significantly fewer physicians (43\%) than patients (84.6\%) were of the opinion that all of the medical orders must be followed to the letter.

Conclusions. Results suggest that BBN was a stressful experience for physicians. It was mostly related to the fear of disrupting the patient's well-being. Low level of effective communication was caused by the insufficiency of BBN skills. Social and cultural aspects also played a role.
\end{abstract}

Keywords: Clinical-Patient Communication, Doctor-Patient Relationship, Breaking Bad News.

\section{Introduction}

Breaking bad news is one of the hardest duties physicians must face during their professional practice [1]. As numerous studies have shown, the level of soft skills demonstrated by clinicians while disclosing unfavorable information is directly proportional to therapeutic results [2]. Proper communication has a substantial impact on the quality of medical influence, increasing the level of trust in doctor-patient relationship, among other things [3]. On one hand, not only is this communication essential in achieving the patient's full coopera- tion during medical treatment and their involvement in the therapeutic process, it also improves the patient's psychological endurance. It results in faster recovery and/or less severe symptoms of the disease. On the other hand, the level of distress, including the intensity of pain and discomfort the patient is experiencing, increases with anxiety and stress-inducing circumstances, which are correlated to the feeling of being ill-informed or confused. The use of soft skills while breaking bad news serves another vital purpose, which is to protect physicians from excessive stress. The feeling of 
duty well performed reinforces one's self-confidence as a professional and helps to prevent the risk of occupational burnout [4].

With our study we tried to establish how exactly bad news is delivered to patients and what amount of stress Polish physicians are under in such situations. What we wanted to find out was how doctors actually cope, seeing that their responsibility is not only the duty of care, but also to teach and socialize students and young physicians professionally. We were interested in the comparison of the physician and patient preferences in the context of mutual influence in the doctor-patient relationship. We concluded it was an important socio-cultural variable, which determines the method of disclosing medical information.

\section{Material and Methods}

The survey was conducted between February and June of 2015 in the University Clinical Centre in Gdansk. It is a clinical hospital functioning at the Medical University of Gdansk. It has been classified under the highest, third level of specialization. According to the Polish Ministry of Health standards, it is a model health, research and training facility.
The first group of respondents consisted of physicians $(n=100)$ from thirteen different clinics: oncology, internal diseases, cardiac surgery, gynecology, obstetrics, and urology. Adult patients of said clinics ( $n$ = 378), fully responsive and being prepared to leave the hospital when the survey was taking place, constituted the second group. The selection of both clinicians and patients meeting the above criteria was random (Table 1).

The data was collected with the help of a survey questionnaire created by an interdisciplinary team of experts specializing in clinical psychology, medical sociology and medical law. The research instrument contained questions about the sources and level of stress connected with the necessity of disclosing unfavorable prospects to patients as well as the evaluation and nature of the doctor-patient relationship. Statistica v.12 software was used for statistical analysis. The respondents' opinions and evaluations were compared with the demographic, health and medical variables. The analysis of the relationship between the discrete variables and the statistical heterogeneity of the respondent groups was made with the use of the Pearson's chi-square test. The differences were considered statistically significant if the $p<0.05$.

Table 1. Characteristics of the Respondents

\begin{tabular}{|c|c|c|c|c|c|}
\hline Doctors & $n$ & $\%$ of $n$ & Patients & $n$ & $\%$ of $n$ \\
\hline Sex & & & Sex & & \\
\hline Women & 52 & 53.6 & Women & 246 & 65.8 \\
\hline Men & 42 & 46.4 & Men & 128 & 34.2 \\
\hline Age & & & Age & & \\
\hline$\leqslant 30$ & 23 & 23.0 & $18-30$ & 46 & 12.3 \\
\hline $31-40$ & 42 & 42.0 & $31-40$ & 55 & 14.7 \\
\hline $41-50$ & 25 & 25.0 & $41-50$ & 65 & 17.4 \\
\hline \multirow[t]{2}{*}{$51-60$} & 10 & 10.0 & $51-60$ & 63 & 16.8 \\
\hline & & & $\geqslant 60$ & 145 & 38.8 \\
\hline Academic degree & & & Marital status & & \\
\hline M.D. & 55 & 60.4 & Single & 57 & 15.2 \\
\hline Ph.D. & 29 & 31.9 & Married & 267 & 71.2 \\
\hline Sc.D. & 7 & 7.7 & Divorced & 24 & 6.4 \\
\hline Specialty & & & Widow / Widower & 27 & 7.2 \\
\hline None & 44 & 45.4 & Education & & \\
\hline One & 23 & 23.7 & Junior high school & 3 & 0.8 \\
\hline Two & 28 & 28.9 & Vocational & 91 & 24.6 \\
\hline Three and more & 2 & 2.1 & Secondary & 119 & 32.2 \\
\hline Position & & & Higher & 135 & 36.5 \\
\hline Junior doctor & 3 & 3.5 & Hospitalization time in a year & & \\
\hline Resident doctor & 31 & 36.5 & $\leqslant 7$ days & 191 & 51.3 \\
\hline Junior assistant & 11 & 12.9 & 8-14 days & 93 & 25.0 \\
\hline Senior assistant & 38 & 44.7 & 15-21 days & 26 & 7.0 \\
\hline Senior registrar & 2 & 2.4 & $\geqslant 22$ days & 62 & 16.7 \\
\hline
\end{tabular}




\section{Results}

Ways of delivering bad news

We began our study with an attempt to determine the physicians' approach to breaking bad news (Table 2). Most of them declared they always inform their patients personally and in full detail about unfavorable medical diagnosis and/or prognosis (66.7\%). More than every tenth clinician admitted they convey only carefully pre-selected information, which means their patients are not being fully informed about their clinical state. Exactly $58 \%$ of the doctors claimed they would be interested in a communication procedure, which could provide some effective methods of disclosing unfavorable news, were it available in Poland.

\section{Levels of stress}

A significant majority of the physicians recognized the moment of delivering bad news as extremely stressful, regardless of the way it was done (Table 3). More than half of the respondents declared intense stressor overload. Specialists described their experiences as highly stressful significantly more often than residents ( $p=$ 0.03929).

\section{Sources of stress}

According to the surveyed clinicians, the fear of depriving their patients of hope was the main (56.1\%) cause of stress (Table 4 ). A substantial percentage (38\%) of respondents admitted they felt uncomfortable knowing patients were expecting to

Table 2. Physicians' approach to breaking bad news

\begin{tabular}{lc}
\hline \multicolumn{1}{|c}{ Categories of response } & $\%$ of $n=100$ \\
\hline I always inform my patients personally and in full detail & 66.7 \\
\hline I do not inform my patients in hope they will figure it out themselves & 0.0 \\
\hline I prefer to disclose unfavorable news only to the patient's family & 1.0 \\
\hline My patients get information from the medical documentation they are given when leaving the hospital & 1.0 \\
\hline I issue a referral for my patient to see a specialist, hoping the information will be given there & 0.0 \\
\hline I convey only pre-selected information & 12.1 \\
\hline I have other methods than mentioned above & 19.2 \\
\hline
\end{tabular}

Table 3. Declarative level of perceived stress

\begin{tabular}{lcc}
\hline \multicolumn{1}{c}{ Level of stress } & $\mathrm{n}=92$ & $\%$ of $n$ \\
\hline No stress & 4 & 4.3 \\
\hline Very low and low level of stress & 15 & 16.3 \\
\hline Moderate level of stress & 25 & 27.2 \\
\hline High and very high level of stress & 46 & 50.0 \\
\hline Maximum stress intensity & 2 & 2.2 \\
\hline
\end{tabular}

* The respondents were asked to indicate their answer on an 11-point scale, where 0 meant "no stress" and 10 meant "maximum stress intensity". The answers were categorized as follows: $0=$ "no stress", 1-3 = "very low and low level of stress", 4-6 = "moderate level of stress", 7-9 = "high and very high level of stress", and $10=$ "maximum stress intensity".

Table 4. Causes of stress involved in breaking bad news

\begin{tabular}{lc}
\hline \multicolumn{1}{c}{ Categories of response* } & $\%$ of $n=83$ \\
\hline Depriving the patient of hope & 56.1 \\
\hline Patient's emotional response & 37.3 \\
\hline Lack of sufficient training & 9.6 \\
\hline Time limit & 14.4 \\
\hline Prognostic uncertainty & 15.7 \\
\hline Family members insisting on nondisclosure of unfavorable information & 4.8 \\
\hline The feeling of inadequacy or hopelessness & 12.0 \\
\hline A long-term relationship with the patient & 4.8 \\
\hline Patient's expectations as to the positive outcome of treatment & 38.5 \\
\hline * The respondents could pick only two of the answers.
\end{tabular}


hear good news about their treatment, and communicating unfavorable information would mean letting them down. Exactly $37.3 \%$ of the doctors disclosed they were afraid of emotional response. Almost every seventh respondent (15.7\%) picked out prognostic uncertainty and the discomfort caused by insufficient amount of time they were able to offer their patients when delivering bad news (14.4\%). Every tenth physician experienced the stress-inducing feeling of inadequacy and hopelessness while delivering unfavorable news. Only $9,6 \%$ of the clinicians recognized lack of training and the resulting skill deficiency in terms of communicating bad news as considerably stress-inducing.

Key aspects of the doctor-patient relationship Clinical communication is substantially determined by cultural and social references. Thus, we asked doctors and patients for evaluation of the main components constituting the physician-patient relationship (Table 5). Majority of respondents from both groups declared they preferred partnership in the doctor-patient interaction. In their opinion, the patient should also have the right to participate in conscious decision-making concerning therapeutic choices. Most of the interviewees recognized the physician's obligation to inform the patient fully about their health. There was a considerable asymmetry as to the issue of following medical orders. A significant majority of patients (84.6\%) believed they must follow all medical orders to the letter. Only $43 \%$ of the clinicians held the same view, while every fourth physician decided that their patients are not obliged to adhere to treatment recommendations.

\section{Discussion}

Numerous statistics show that delivering unfavorable information is a highly stressful task for physicians [1, 5-7]. As can be seen from the results of our survey, Polish doctors face the same problem. More than 52\% of the clinicians admitted they felt intensely stressed while disclosing bad news (see Table 3). In our opinion, however, these results need to be interpreted with the socio-cultural aspect in mind. Even though it affects the doctor-patient relationship noticeably [8], it is rarely taken into consideration during research. We believe that the principle of autonomy, fundamental in the Anglo-Saxon countries, translates into how physicians understand their duty of delivering unfavorable information. Suitable preparation for this task, offered to future clinicians as part of medical education [9], notification protocols [10-12] and psychological support, are further elements of importance owing to their stress-reducing function.

Our study has shown that, in case of Polish doctors, basic stress-inducing categories connected to breaking bad news involve anxieties concerning the patient's well-being (fear of depriving the patient of hope or being unable to meet the patient's therapeutic expectations; see Table 4). Although the majority of both patients and clinicians have declared they prefer partnership in doctor-patient interactions, the percentage of neutral opinions has also been sizeable. As many as $40 \%$ of the surveyed patients expressed their negative view about the idea of physician-patient therapeutic partnership (see Table 5). The paternalistic model of practice seems to remain deeply rooted not only as a physicians' attitude but also as some patients' expectation.

Table 5. Comparison of respondents' preferences as to key aspects of the doctor-patient relationship

\begin{tabular}{|c|c|c|c|c|}
\hline \multirow{2}{*}{\multicolumn{2}{|c|}{ Evaluative statement }} & Disagree* $^{*}$ & Neutral* $^{*}$ & Agree $^{*}$ \\
\hline & & \multicolumn{3}{|c|}{$n(\%)$} \\
\hline \multirow{2}{*}{ Patient and physician are partners in the therapeutic process } & Doctors & $6(6.0)$ & $39(39.0)$ & $55(55.0)$ \\
\hline & Patients & $40(11.6)$ & $74(21.4)$ & $231(67.0)$ \\
\hline \multirow{2}{*}{ Patients must follow all medical orders to the letter } & Doctors & $25(25.0)$ & $32(32.0)$ & $43(43.0)$ \\
\hline & Patients & $15(4.3)$ & $39(11.1)$ & $297(84.6)$ \\
\hline \multirow{2}{*}{ Patients have the right to participate in conscious decision-making about their own health } & Doctors & $2(2.0)$ & $10(10.0)$ & $88(88.0)$ \\
\hline & Patients & $8(2.3)$ & $23(6.6)$ & $318(91.1)$ \\
\hline \multirow{2}{*}{ Physicians are obliged to disclose all information concerning the patient's health to the patient } & Doctors & $2(2.0)$ & $18(18.0)$ & $80(80.0)$ \\
\hline & Patients & $8(2.3)$ & $15(4.3)$ & $329(93.4)$ \\
\hline
\end{tabular}

* The respondents were asked to indicate their answer on an 6-point scale, where 1 "I strongly disagree" and 2 "I disagree" were put into the "I disagree" category, whereas 6 "I strongly agree" and 5 "I agree" were categorized as "I agree". Answers 3 and 4 were neutral and as such they were placed in the "Neutral" category. 
The basic rule of the Polish medical model of ethics is the commitment to the priority of the patient's well-being. Any news which may disrupt it may not be delivered should the physician, in their subjective certainty, find its predictable consequences iatrogenic. Article 17 of the Polish Medical Code of Ethics includes the following guideline: "Information about diagnosis and unfavorable prognosis may not be disclosed to the patient only if the physician strongly believes that such disclosure will cause the patient great harm or affect the patient's health negatively in any other way; should the patient, however, explicitly demand otherwise, full information ought to be given". Put into practice, it means that clinicians with especially low level of soft skills and experiencing chronic stress tend to use the exception described in Article 17 to justify nondisclosure of information or to communicate only its pre-selected, shortened version.

The way bad news are delivered is another issue. High stress intensity is directly related to faulty clinical decision-making and results in premature closure [6]. Notification protocols may offer a satisfactory solution here. Unlike Anglo-Saxon countries, where these are considered standard, Polish educational system is not widely familiar with communication procedures of this kind [13]. As of today, basic communication skills shaping courses still have not found their place among regular academic modules offered to medical students in Poland. If they exist in any form, it is rudimentary and rather theoretical. Practical training of soft skills is hence available solely with the help of commercial courses organized outside of universities, i.e., hospices, private institutions, foundations, and associations. Over the course of the years merely two protocols have been created which may be considered useful in academic training and competence development. "The 5 Steps Method" is a procedure for communicating news about the death or a serious illness of a child to the parents [14]. "EMPATHY” is a protocol for disclosing unfavorable information to the parents of oncological patients [15]. No procedure has been established with adult patients in mind, even though almost six in ten clinicians would be interested in using it, as the results of our study have shown. It is true that clinical psychologists are being hired more and more often to assist doctors in breaking bad news. It is still not common practice, however. Furthermore, psychological services are provided exclusively to the patients. Formally, Polish physicians do not receive any support when coping with difficult clinical situations.
Those key factors seem to contribute to the low quality of clinical communication in general. In consequence, Polish doctors have ranked the lowest among all of the 18 countries participating in OECD research [16] in all of the categories, (1) "Spending enough time with patient" maximum: Belgium 97.5, OECD18 84.9, USA 80.9, minimum: Poland 69.6,; (2) "Easy-to-understand explanations" maximum: Belgium 97.8, OECD18 87.9, USA 86.3, Poland 69.6; (3) "Giving opportunity to ask questions or raise concerns" maximum: Belgium 97.7, OECD18 85.0, USA 86.7, minimum: Poland 33.6; (4) "Involving patient in decisions about care and treatment" maximum: Luxembourg 95.4, OECD18 81.3, USA 83.9, minimum: Poland 47.9. It should be emphasized that, according to the OECD report, Poland holds third place in regard to the number of consultations provided by doctors per person per year. The organization of the health care system also seems to be an important variable, as it limits clinicians with excessive bureaucracy, hence reducing their time for direct contact with patients.

Through this study a number of issues emerged surrounding contraceptive method decision-making that could inform development of messaging and policy changes. First, communication campaigns could work to de-mystify the process that health 15 professionals use to support contraceptive decision-making. Communication campaigns should also help clients understand their important role in method choice by increasing their internal locus of control about contraceptive method decision-making. These campaigns would work best if done in tandem with training among contraceptive providers on client-centered counseling, including the important role of clients in the selection of the contraceptive method. Second, given how important switching contraceptive methods is in response to unmanageable side effects, contraceptive providers should be trained to discuss the strategy of switching to all clients - potential future clients, new clients, and continuing clients. Third, all persons who provide contraceptive methods, including those in the private sector, would benefit from training on client-centered counseling, especially related to counseling all clients - new and returning - on potential side effects. Widely disseminating accurate information about the importance of individual preference in contraceptive method choice, and the ability to switch methods, could increase contraceptive use in Nigeria through increased use among non-users, satisfaction with use among current users, and the power that comes from feeling in control. 
As our study has shown, BBN was an intensely stressful experience for Polish physicians. This can be largely attributed to the fear of BBN disrupting the patient's well-being. Low level of soft competencies is, in our opinion, only one of the reasons for such an attitude. It has also a lot to do with paternalism, still present in some form and visible in the patients' expectations. Thus, we suggest that physician-targeted educational content should include notification protocols for BBN as part of soft skills shaping training programs. Even if the aforementioned tools (e.g. SPIKES protocol), which are created in Anglo-Saxon countries, happen to be less applicable for the other European patients [17], we do believe the above suggestion is valid, considering the low quality indicators of clinical communication in Poland. System solutions, such as communication skills training courses and psychological assistance for physicians experiencing extreme stress, also require due support.

\section{Acknowledgements}

\section{Conflict of interest statement}

The authors declare no conflict of interest.

\section{Funding sources}

There are no sources of funding to declare.

\section{Informed consent and ethical approval}

Informed consent was obtained from all individual participants included in the study. The research was positively evaluated and approved by the Independent Bioethics Commission for Research at the Medical University of Gdansk.

\section{References}

1. Hulsman RL, Pranger S, Koot S, Fabriek M, Karemaker JM, Smets EM. How stressful is doctor-patient communication? Physiological and psychological stress of medical students in simulated history taking and bad-news consultations. Int J Psychophysiol. 2010;77:26-34. doi:10.1016/j.jpsycho.2010.04.001.

2. Zolnierek KB, Dimatteo MR. Physician communication and patient adherence to treatment: a meta-analysis. Med Care. 2009;47:826-34. doi:10.1097/ MLR.0b013e31819a5acc.

3. Levinson W, Hudak P, Tricco AC. A systematic review of surgeon-patient communication: strengths and opportunities for improvement. Patient Educ Couns. 2013;93:317. doi:10.1016/j.pec.2013.03.023.

4. Ramirez AJ, Graham J, Richards MA, Cull A, Gregory WM. Mental health of hospital consultants: The effects of stress and satisfaction at work. Lancet. 1996;347:724-8. doi:10.1016/S0140-6736(96)90077-X.

5. Cohen L, Baile WF, Henninger E, Agarwal SK, Kudelka AP, Lenzi $R$, et al. Physiological and psychological effects of delivering medical news using a simulated physician-patient scenario. J Behav Med. 2003;26:459-71.

6. Pottier P, Dejoie T, Hardouin JB, Le Loupp AG, Planchon $B$, Bonnaud A, et al. Effect of stress on clinical reasoning during simulated ambulatory consultations. Med Teach. 2013;35:472-80. doi:10.3109/0142159X.2013.774336.

7. Shaw J, Brown R, Heinrich P, Dunn S. Doctors' experience of stress during simulated bad news consultations. Patient Educ Couns. 2013;93:203-8. doi:10.1016/j. pec.2013.06.009.

8. Street RL, Krupat E, Bell RA, Kravitz RL, Haidet P. Beliefs about control in the physician-patient relationship: effect on communication in medical encounters. J Gen Intern Med. 2003;18:609-16.

9. Hargie O, Dickson D, Boohan M, Hughes K. A survey of communication skills training in UK schools of medicine: present practices and prospective proposals. Med Educ. 1998;32:25-34.

10. Baile WF, Buckman R, Lenzi R, Glober G, Beale EA, Kudelka AP. SPIKES-A six-step protocol for delivering bad news: application to the patient with cancer. Oncologist. 2000;5:302-11.

11. Fine RL. Personal choices--communication among physicians and patients when confronting critical illness. Tex Med. 1991;87:76-82.

12. Rabow MW, McPhee SJ. Beyond breaking bad news: how to help patients who suffer. West J Med. 1999;171:260-3.

13. Sobczak K. Communicating bad news about the diagnosis and prognosis. Forum Med Rodz. 2014;8:203-8.

14. Barton-Smoczyńska I. Method of five steps. Informing the parents that their child has fallen ill or died during pregnancy. Życie i Płodność. 2009;4:34-49.

15. Jankowska KA. Communication in pediatric oncology. Disclosing a cancer diagnosis. Psychoonkologia. 2014;77:123-7.

16. Health at a Glance 2015. 2015.

17. Seifart C, Hofmann M, Bär T, Riera Knorrenschild J, Seifart $\mathrm{U}$, Rief $\mathrm{W}$. Breaking bad news-what patients want and what they get: evaluating the SPIKES protocol in Germany. Ann Oncol. 2014;25:707-11. doi:10.1093/annonc/ mdt582.

Acceptance for editing: 2016-06-10 Acceptance for publication: 2016-06-23

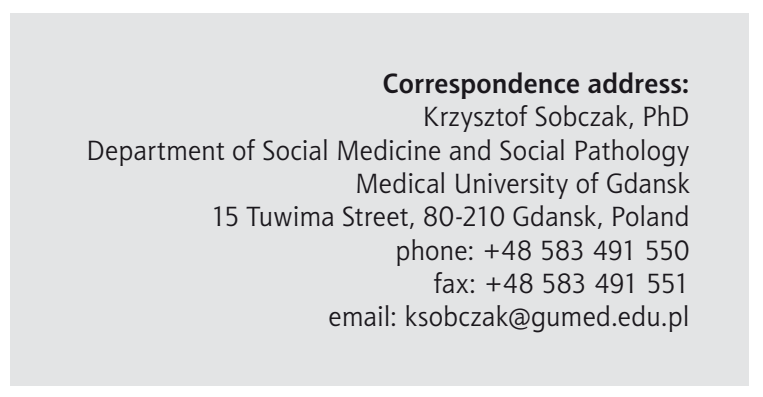

